\title{
Monitoring Response to Transarterial Chemoembolization in Hepatocellular Carcinoma Using ${ }^{18}$ F-Fluorothymidine PET
}

\author{
Rohini Sharma ${ }^{1}$, Marianna Inglese ${ }^{1}$, Suraiya Dubash ${ }^{1}$, Haonan Lu ${ }^{1}$, David J. Pinato ${ }^{1}$, Chandan Sanghera ${ }^{1}$, Neva Patel ${ }^{1,2}$, \\ Anthony Chung ${ }^{1}$, Paul D. Tait ${ }^{3}$, Francesco Mauri ${ }^{1}$, William R. Crum ${ }^{1,4}$, Tara D. Barwick ${ }^{1,3}$, and Eric O. Aboagye ${ }^{1}$ \\ ${ }^{I}$ Department of Surgery and Cancer, Imperial College London, Hammersmith Hospital, London, United Kingdom; ${ }^{2}$ Radiological \\ Sciences Unit, Imperial College Healthcare NHS Trust, London, United Kingdom; ${ }^{3}$ Department of Radiology, Imperial College \\ Healthcare NHS Trust, London, United Kingdom; and ${ }^{4}$ Institute of Translational Medicine and Therapeutics, Imperial College \\ London, London, United Kingdom
}

\begin{abstract}
Accurate disease monitoring is essential after transarterial chemoembolization (TACE) in hepatocellular carcinoma (HCC) because of the potential for profound adverse events and large variations in survival outcome. Posttreatment changes on conventional imaging can confound determination of residual or recurrent disease, magnifying the clinical challenge. On the basis of increased expression of thymidylate synthase (TYMS), thymidine kinase $1(T K-1)$, and equilibrative nucleoside transporter 1 (SLC29A1) in HCC compared with liver tissue, we conducted a proof-of-concept study evaluating the efficacy of $3^{\prime}$-deoxy-3'-18 F-fluorothymidine ( $\left.{ }^{18} \mathrm{~F}-\mathrm{FLT}\right)$ PET to assess response to TACE. Because previous PET studies in HCC have been hampered by high background liver signal, we investigated whether a temporal-intensity voxel clustering (kinetic spatial filtering, or KSF) improved lesion detection. Methods: A tissue microarray was built from $36 \mathrm{HCC}$ samples and from matching surrounding cirrhotic tissue and was stained for $T K-1$. A prospective study was conducted; 18 patients with a diagnosis of HCC by the criteria of the American Association for the Study of Liver Diseases who were eligible for treatment with TACE were enrolled. The patients underwent baseline conventional imaging and dynamic ${ }^{18} \mathrm{~F}-\mathrm{FLT}$ PET with KSF followed by TACE. Imaging was repeated 6-8 wk after TACE. The PET parameters were compared with modified enhancement-based RECIST. Results: Cancer Genome Atlas analysis revealed increased RNA expression of TYMS, TK-1, and SLC29A1 in HCC. TK-1 protein expression was significantly higher in HCC $(P<0.05)$. The sensitivity of ${ }^{18} \mathrm{~F}-\mathrm{FLT}$ PET for baseline HCC detection was $73 \%$ (SUV $_{\max }$, $9.7 \pm 3.0$; tumor to liver ratio, $1.2 \pm 0.3$ ). Application of KSF did not improve lesion detection. Lesion response after TACE by modified RECIST was $58 \%$ (14 patients with 24 lesions). A $30 \%$ reduction in mean ${ }^{18}$ F-FLT PET uptake was observed after TACE, correlating with an observed PET response of $60 \%$ (15/25). A significant and profound reduction in the radiotracer delivery parameter $K_{1}$ after TACE was observed. Conclusion: ${ }^{18} \mathrm{~F}-\mathrm{FLT}$ PET can differentiate HCC from surrounding cirrhotic tissue, with PET parameters correlating with TACE response. KSF did not improve visualization of tumor lesions. These findings warrant further investigation.
\end{abstract}

Received Dec. 8, 2019; revision accepted Apr. 3, 2020

For correspondence or reprints contact: Rohini Sharma, Imperial College London, Hammersmith Campus, Du Cane Rd., W12 OHS, London, U.K.

E-mail: r.sharma@imperial.ac.uk

Published online Jun. 8, 2020.

Immediate Open Access: Creative Commons Attribution 4.0 International License (CC BY) allows users to share and adapt with attribution, excluding materials credited to previous publications. License: https://creativecommons. org/licenses/by/4.0/. Details: http://jnm.snmjournals.org/site/misc/permission. xhtml.

COPYRIGHT @ 2020 by the Society of Nuclear Medicine and Molecular Imaging.
Key Words: ${ }^{18} \mathrm{~F}-\mathrm{FLT}$ PET; hepatocellular cancer; response

J Nucl Med 2020; 61:1743-1748

DOI: 10.2967/jnumed.119.240598

$\mathbf{T}$ he recommended treatment option for intermediate-stage hepatocellular carcinoma (HCC) is transarterial chemoembolization (TACE), which involves the delivery of a cytotoxic agent commonly mixed with lipiodol followed by selective embolization of the tumoral arterial supply (1). The typical vascular pattern of HCC on contrast-enhanced CT or MRI is early arterial enhancement followed by washout. Although both contrast-enhanced CT and MRI are widely used to assess response after TACE, there is uncertainty in their ability to detect viable disease after TACE (2). Modified RECIST (mRECIST), which measures changes in arterial enhancement as a marker of residual viable tumor, is a more accurate measure of tumor response to treatment than standard RECIST and is routinely used in the assessment of HCC (3). However, lipiodol deposition can induce beam-hardening artifacts and obscure enhancement in the arterial phase, reducing the sensitivity of CT after TACE. With MRI, coagulative hemorrhagica necrosis may lead to a high T1 signal, making it difficult to assess enhancement (4).

PET imaging has been evaluated in HCC for staging and response assessment (5). Studies investigating ${ }^{18} \mathrm{~F}-\mathrm{FDG}$ in $\mathrm{HCC}$ show limited sensitivity $(50 \%-70 \%)$ due to similar activities of glycolytic enzymes and glucose 6-phosphatase in liver and welldifferentiated $\mathrm{HCC}$, resulting in near-equivalent uptake of ${ }^{18} \mathrm{~F}-\mathrm{FDG}$ (6). In imaging with single-agent ${ }^{11} \mathrm{C}$-acetate and ${ }^{11} \mathrm{C}$-choline, ${ }^{18} \mathrm{~F}$ choline are similarly limited, culminating in the exploitation of dualtracer techniques to improve sensitivity and specificity (7).

$3^{\prime}$-deoxy- $3^{\prime}-{ }^{18} \mathrm{~F}$-fluorothymidine $\left({ }^{18} \mathrm{~F}\right.$-FLT $)$ is a surrogate marker of proliferation, with uptake reflecting the activity of thymidine kinase $1(T K-1)$, whose expression correlates with ex vivo proliferation biomarkers (8). Unlike ${ }^{18} \mathrm{~F}$-FDG, the uptake of ${ }^{18} \mathrm{~F}$-FLT is more specific for proliferation and is unaffected by inflammation, a particular concern because HCC tumors develop within a proinflammatory milieu (9). To date, a single study of ${ }^{18}$ F-FLT PET in HCC indicated that $69 \%$ of patients had uptake higher than background liver whereas the remaining lesions were either photopenic or of mixed uptake (10). However, the patient group was heterogeneous, including cholangiocarcinoma, and no information was given about therapy response. To improve lesion detection, we have 
previously applied a temporal voxel-clustering approach-kinetic spatial filtering (KSF) - for removing normal, physiologic hepatic ${ }^{18}$ F-FLT uptake and to visualize specific uptake (i.e., uptake due to phosphorylation) in liver metastases (11). Briefly, the KSF compares the time-activity curves of each voxel with the time-activity curve of predefined tissue classes such as liver and tumor. Voxels classed as liverlike are excluded, thereby removing areas of physiologic uptake unrelated to ${ }^{18}$ F-FLT retention.

This study evaluated the clinical utility of ${ }^{18}$ F-FLT PET in assessing TACE response in HCC. We first reviewed the RNA expression of key targets in the metabolism of ${ }^{18} \mathrm{~F}$-FLT using large published datasets of HCC. We then investigated the tissue expression of $T K-1$ in HCC and surrounding cirrhosis, an important consideration in developing a tracer paradigm that will effectively differentiate cirrhotic tissue from HCC. Finally, we undertook a prospective study using dynamic ${ }^{18}$ F-FLT PET both to visualize the tumors and to use as a response biomarker, incorporating application of the KSF and kinetic modeling.

\section{MATERIALS AND METHODS}

\section{Bioinformatics Analysis}

An RNA-sequencing dataset containing $371 \mathrm{HCC}$ and 50 nonmalignant tissue samples from The Cancer Genome Atlas project was measured from the Illumina HiSEquation 2000 RNA Sequencing platform. The RNA-Seq by expectation maximization-normalized data were downloaded from UCSC Xena (http://xena.ucsc.edu/). Differential gene expression of TK1, thymidylate synthase (TYMS), and equilibrative nucleoside transporter 1 (SLC29A1) comparing tumor and nonmalignant tissue was performed using the ggplot2 package and the t.test function in $\mathrm{R}$, version 3.5.2.

\section{Tissue Microarray}

Immunohistochemistry for $T K-1$ (1:100; AbCam) was performed on a tissue microarray from 36 patients with a histologic diagnosis of HCC. A trained histopathologist who was unaware of the clinical data scored all cases manually using the immunohistochemical score (12). Access to retrospective tissue specimens was granted by the Imperial College Tissue Bank (approval R16005).

\section{Prospective Study Design}

Eighteen patients with HCC were prospectively enrolled (Supplemental Appendix 1; supplemental materials are available at http:// jnm.snmjournals.org). Patients received standard TACE with liposomal doxorubicin emulsified in lipiodol followed by embolization with gelatin sponge particles. Baseline staging included contrast-enhanced CT or MRI of the liver $28 \mathrm{~d}$ before TACE; the same imaging modality was repeated 6-8 wk after TACE to evaluate treatment response, followed by 3 monthly until disease progression. mRECIST for HCC (13) was documented by a single experienced hepatobiliary radiologist.

\section{Image Analysis}

Lesions on ${ }^{18}$ F-FLT PET corresponding to those larger than $10 \mathrm{~mm}$ on CT or MRI and showing increased uptake were considered target lesions and used for analyses both on PET/CT and on CT or MRI before and after treatment.

Consecutive regions of interest were manually defined on the summed images and encompassed the whole tumor for SUV analysis. The ${ }^{18} \mathrm{~F}$-FLT radioactivity concentration within the regions of interest was normalized for injected radioactivity and body weight (grams) to obtain $\mathrm{SUV}_{\text {mean }}$ and $\mathrm{SUV}_{\text {max }}$ at 60 min $\left(\mathrm{SUV} 0_{\text {mean }}\right.$ and $\mathrm{SUV60}$ max, respectively) on baseline and posttreatment ${ }^{18} \mathrm{~F}-\mathrm{FLT}$ PET/CT studies. The percentage change in both $\mathrm{SUV}_{\text {mean }}$ and $\mathrm{SUV}_{\text {max }}$ was calculated for each target lesion visible on baseline imaging as (posttreatment SUV - baseline SUV)/baseline SUV. In each case, a 3-cm region of interest was placed in the liver in a tumor-free area to measure background liver $\mathrm{SUV}_{\text {mean }}$, and the ratio of tumor $\mathrm{SUV}_{\max }$ to liver $\mathrm{SUV}_{\text {mean }}$ was determined.

\section{Quantitative Analysis}

A metabolite-corrected image-derived arterial input function was implemented during kinetic modeling of data with a 2-tissue-compartment model.

\section{Statistical Analysis}

Because this was a pilot study, no formal power calculation was undertaken. Summary statistics of the associations between PET parameters and clinical outcome were determined. Because of the small sample size, patients were grouped as responders (complete or partial response) or nonresponders (stable or progressive disease). The relationship between kinetic parameters and response was evaluated using Wilcoxon rank tests. The $\chi^{2}$ test was used to evaluate the utility of the tracer before and after TACE therapy. Concordance was determined using Cohen $\kappa$-analysis. A $P$ value of 0.05 or less was considered significant. All statistical analyses were conducted using the SPSS statistical package, version 22 (SPSS Inc.).

\section{RESULTS}

\section{Increased Expression of Thymidine Metabolism Enzymes in $\mathrm{HCC}$}

Using RNA-sequencing data from The Cancer Genome Atlas, we observed a significantly higher expression of TYMS, TK-1, and SLC29A1 in tumor tissue $(n=371)$ than in adjacent nonmalignant tissue $(n=50$; $P<0.05$ ) (Supplemental Figs. 1A-1C). Using a tissue microarray, we observed a significantly higher $T K-1$ expression in HCC tumors (median immunohistochemical score, 33; range, 0-300) than in the surrounding parenchyma (score, 0; range, 0-50), suggesting that ${ }^{18} \mathrm{~F}$-FLT has the potential to differentiate HCC from surrounding cirrhotic liver $(P=0.004)$ (Supplemental Figs. 2A-2C).

\section{Patient Characteristics}

Eighteen patients were enrolled (16 men and 2 women), with 16 patients completing the study (Table 1). The median age was $68 \mathrm{y}$ (range, 42-79 y). All patients received TACE for intermediatestage disease. Three patients had received TACE previously and were undergoing retreatment for residual active disease; the remaining patients were treatment-naïve.

\section{Visibility of HCC Above Background on ${ }^{18} \mathrm{~F}-\mathrm{FLT}$ PET Images}

Twenty-six liver lesions (median size, $29.5 \mathrm{~mm}$; range, 10$117 \mathrm{~mm}$ ) were identified on conventional imaging; 5 had baseline MRI and the remainder CT. On visual analysis of the PET images, 19 lesions had a level of uptake above that of the background liver (73\% sensitivity) (Figs. 1A-1F).

All lesions were included in the analysis. The mean SUV60 mean $( \pm \mathrm{SD})$ and $\mathrm{SUV}_{\mathrm{max}}$ on baseline imaging were $6.5 \pm 1.9$ and $9.7 \pm 3.0$, respectively. The SUV60 mean of the background liver was $6.1 \pm 0.9$. A significant difference was observed between the SUV60 $0_{\max }$ of the cancer and that of the surrounding, noncancerous, liver tissue $(P=0.02)$, with a tumor-to-liver ratio of $1.2 \pm 0.3$, confirming that uptake in HCC was above cirrhotic background activity, enabling visualization on ${ }^{18}$ F-FLT PET scans in most cases.

\section{No Improvement of Visualization upon KSF}

Background liver activity was completely filtered out in 12 of 16 patients; 4 patients retained partial background liver activity. KSF did not improve image visualization above that of PET/CT imaging; 11 of the 26 lesions (42\%) were visible after application of KSF, compared with 19 lesions without KSF (Figs. 2A-2F). Small 
TABLE 1

Imaging Features of HCC Lesions

\begin{tabular}{|c|c|c|c|c|c|c|c|c|c|c|}
\hline \multirow{2}{*}{$\begin{array}{c}\text { Patient } \\
\text { no. }\end{array}$} & \multirow{2}{*}{$\begin{array}{l}\text { Lesion } \\
\text { location }\end{array}$} & \multirow{2}{*}{$\begin{array}{l}\text { Lesion } \\
\text { size } \\
(\mathrm{mm})\end{array}$} & \multirow{2}{*}{$\begin{array}{l}\text { Uptake above } \\
\text { background } \\
\text { (visual) }\end{array}$} & \multirow{2}{*}{$\begin{array}{l}\text { Background } \\
\text { SUV }_{\text {mean }}\end{array}$} & \multicolumn{2}{|c|}{$\begin{array}{l}\text { SUV60 } \times \\
10^{-5} \mathrm{~m}^{2} \mathrm{~mL}^{-1} \\
\text { before } R x\end{array}$} & \multirow{2}{*}{$\begin{array}{l}\mathrm{mRECIST} \\
\text { response }\end{array}$} & \multicolumn{2}{|c|}{$\begin{array}{c}\text { Percentage } \\
\text { change in SUV60 }\end{array}$} & \multirow{2}{*}{$\begin{array}{l}\text { Progression- } \\
\text { free survival } \\
\text { (mo) }\end{array}$} \\
\hline & & & & & Mean & Maximum & & Mean & Maximum & \\
\hline 1 & $\begin{array}{l}\text { Diffuse } \\
\text { disease, } \\
R \text { lobe }\end{array}$ & 117 & Isotense & 4.3 & 3.4 & 5.1 & PR & -12.2 & 13.9 & 3 \\
\hline 2 & Segment V/VI & 56 & $\begin{array}{l}\text { Hyperintense rim } \\
\text { and hypointense } \\
\text { center }\end{array}$ & 4.9 & 4.3 & 7.8 & PR & -55.5 & -45.5 & 6.6 \\
\hline 3 & Segment VII & 60 & Isotense & 6.3 & 5.7 & 7.9 & $\mathrm{SD}$ & -65.2 & -42.6 & 5.3 \\
\hline 4 & Segment VI & 20 & Isotense & 5.5 & 2.8 & 5.5 & SD & -6.0 & -14.4 & 4.5 \\
\hline 4 & Segment V & 52 & Isotense & 5.5 & 4.1 & 5.8 & SD & -0.9 & -6.7 & \\
\hline \multirow[t]{7}{*}{5} & Segment II & 44 & Hyperintense & 6.6 & 7.5 & 10.3 & $\mathrm{SD}$ & -37.4 & 1.4 & 11.4 \\
\hline & Segment VI* & 24 & Hyperintense & 6.6 & 7.6 & 9.9 & $\mathrm{SD}$ & 10.9 & -0.7 & \\
\hline & Segment IV & 19 & Hyperintense & 6.6 & 7.3 & 9.8 & SD & 15.5 & 26.2 & \\
\hline & Segment IV & 16 & Hyperintense & 6.6 & 7.8 & 10.3 & $\mathrm{SD}$ & 5.9 & 1.9 & \\
\hline & Segment VIII & 15 & Hyperintense & 6.6 & 7.1 & 9.4 & SD & 0.07 & 0.9 & \\
\hline & Segment IV & 13 & Hyperintense & 6.6 & 7.8 & 11.3 & $\mathrm{SD}$ & -4.7 & 0.9 & \\
\hline & Segment VIII & 19 & Hyperintense & 6.6 & 7.1 & 8.2 & PR & 22.2 & 36.5 & \\
\hline 6 & Segment III & 22 & Hyperintense & 4.8 & 7.6 & 10.2 & NE & -69.2 & -61.9 & 17.3 \\
\hline 7 & Segment III & 22 & Hyperintense & 6.1 & 10.9 & 14.3 & NE & NE & NE & 8.0 \\
\hline 8 & Segment VIII & 42 & Hyperintense & 6.5 & 7.8 & 11.5 & PR & -66.4 & -41.5 & 1.5 \\
\hline 9 & Segment II & 28 & Hyperintense & 6.3 & 7.9 & 10.3 & $\mathrm{CR}$ & -58.6 & -46.1 & 5.2 \\
\hline 10 & Segment I & 62 & Hyperintense & 7.7 & 8.1 & 20.4 & PR & -71.3 & -41.0 & $1.1^{\dagger}$ \\
\hline 11 & Segment III & 38 & Hyperintense & 6.1 & 7.1 & 10.6 & PR & -29.0 & -21.5 & 7.8 \\
\hline 12 & Segment VIII & 59 & $\begin{array}{l}\text { Mixed isotense } \\
\text { and hypotense }\end{array}$ & 8.0 & 5.1 & 10.9 & PR & -55.8 & -32.8 & 16.7 \\
\hline 12 & Segment IV $¥$ & 60 & Hypotense & 8.0 & 2.9 & 8.4 & PR & -51.6 & -33.9 & \\
\hline 13 & Segment VI & 31 & Hyperintense & 4.8 & 6.4 & 8.9 & CR & -39.4 & -48.6 & 19.1 \\
\hline 14 & $\begin{array}{l}\text { Segment VII } \\
\text { (sagittal) }\end{array}$ & 18 & Hyperintense & 5.3 & 5.3 & 7.4 & $\mathrm{CR}$ & -33.9 & -30.5 & 8.1 \\
\hline 14 & Segment VIII & 26 & Hyperintense & 5.3 & 6.3 & 7.5 & CR & -33.5 & -39.1 & \\
\hline 14 & $\begin{array}{l}\text { Segment VII } \\
\text { (medial) }\end{array}$ & 10 & Hyperintense & 5.3 & 6.6 & 8.0 & SD & 17.2 & 30.9 & \\
\hline 15 & Segment VIII & 34 & Hyperintense & 5.5 & 7.3 & 10.4 & PR & -52.4 & -46.3 & 8.9 \\
\hline 16 & Segment VI/VII & 73 & Hyperintense & 5.7 & 6.6 & 11.6 & PR & -65.8 & -24.1 & $14.5 \pi$ \\
\hline $\begin{array}{l}{ }^{*} \text { Untreat } \\
{ }^{\dagger} \text { Patient } \\
{ }^{\ddagger} \text { Photop } \\
\text { I Patient } \\
\text { Rx = tre }\end{array}$ & $\begin{array}{l}\text { d lesion. } \\
\text { died of unrelated } \\
\text { unic lesion. } \\
\text { anderwent liver t } \\
\text { atment; } P R=p a\end{array}$ & $\begin{array}{l}\text { illness. } \\
\text { tial respo }\end{array}$ & tion. & ise; $N E=n$ & - & able; $\mathrm{CR}=$ & lete respc & & & \\
\hline
\end{tabular}

lesions typically had a homogeneous appearance, whereas larger lesions were characterized by perilesional tracer uptake with no measurable ${ }^{18} \mathrm{~F}-\mathrm{FLT}$ trapping in the necrotic center of the tumor. Of the 15 lesions not visualized through KSF, 9 (60\%) were smaller than $30 \mathrm{~mm}$; 3 lesions had higher tissue activity than the HCC average predetermined by the KSF, and the remaining lesions did not retain radiotracer after application of the KSF. Because KSF is associated with removal of delivery components within the data, there was a mean signal reduction of $81 \%$ in the tumors at baseline (range, $18 \%-$
$100 \%$ ) relative to the unfiltered images. The mean reduction in background activity in the liver was $98 \%$ (range, $83 \%-100 \%$ ), resulting in an improved tumor-to-liver ratio of $11.1 \pm$ 17.7.

\section{${ }^{18}$ F-FLT Uptake Parameters and Clinical Outcome}

In terms of response to TACE according to mRECIST, 24 lesions were assessable and 2 were not (1 patient withdrew consent after baseline PET). A response was observed in 14 of the 24 lesions (58\%); no response was observed in the other 10 (42\%). There 


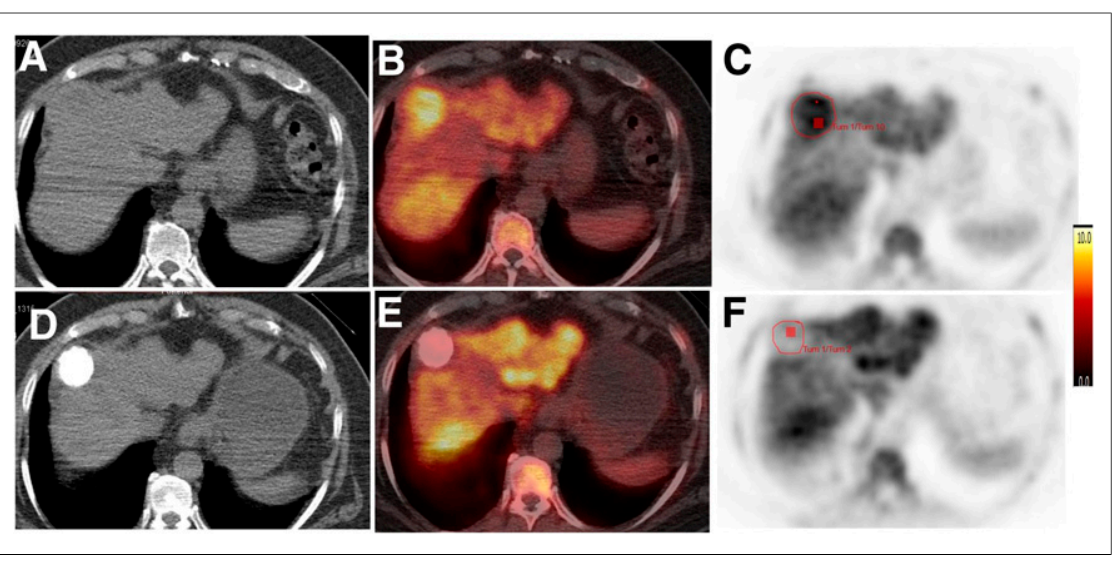

FIGURE 1. Axial CT, ${ }^{18}$ F-FLT PET/CT, and ${ }^{18} \mathrm{~F}$-FLT PET images before (A-C) and after (D-F) TACE show focal HCC lesion (red outline) with increased uptake at baseline and reduction after TACE. Tum = tumor.
$(P<0.001)$. This reduction is consistent with the abrupt cessation of blood flow to the tumor after embolization, resulting in reduced transport of ${ }^{18} \mathrm{~F}$-FLT to the tumor. Although all tumors showed some degree of reduction in $K_{1}$, the change was greater in responders $(66 \%)$ than in nonresponders (50\%) $(P=0.03)$ (Supplemental Fig. 3A). Baseline SUV60 mean and metabolic flux constant $\left(K_{\mathrm{i}}\right)$ were significantly correlated (Pearson $r=0.5, P=0.04$ ), and a significant difference in $K_{\mathrm{i}}$ was observed between baseline imaging $(0.09 \pm 0.03 \mathrm{~mL} / \mathrm{min} / \mathrm{g})$ and post-TACE imaging $(0.04 \pm 0.02 \mathrm{~mL} /$ $\mathrm{min} / \mathrm{g})(P<0.001)$. Baseline $K_{\mathrm{i}}$ and fractional blood volume $\left(\mathrm{V}_{\mathrm{b}}\right)$ were greater in responders than in nonresponders (Supplemental Figs. 3B and 3C) $(P<0.05)$. was a median overall reduction in $\mathrm{SUV}_{\text {mean }}(-29.5 \% \pm$ $31.4 \%)$ and $\mathrm{SUV} 60_{\max }(-18.5 \% \pm 27.5 \%)$ after TACE. Previous test-retest reproducibility studies in breast cancer considered changes in ${ }^{18} \mathrm{~F}$-FLT SUV of more than $20 \%$ as significant (SD, $10 \%-15 \%$ ) (14). Using a $20 \%$ reduction in SUV60 mean to define a PET response led to categorization as lesional response in $60 \%$ (15/ $25)$ and nonresponse in $40 \%$ (10/25). Using Cohen $\kappa$-measures, there was good concordance between lesional PET response and lesional mRECIST $(\kappa=0.66 ; P<0.001 ; 95 \%$ confidence interval, 0.35-0.97).

\section{Kinetic Modeling Evidence of Significant Reduction in Uptake and Retention After TACE}

The analysis of ${ }^{18} \mathrm{~F}$-FLT dynamic data with a 2-tissue-compartment model resulted in physiologically relevant kinetic parameters $(n=14)$ (Table 2$)(15)$. There was a significant mean reduction in the radiotracer delivery parameter $K_{1}$ between baseline $(0.3 \pm 0.1 \mathrm{~mL} / \mathrm{min} / \mathrm{g})$ and after treatment $(0.13 \pm 0.05 \mathrm{~mL} / \mathrm{min} / \mathrm{g})$

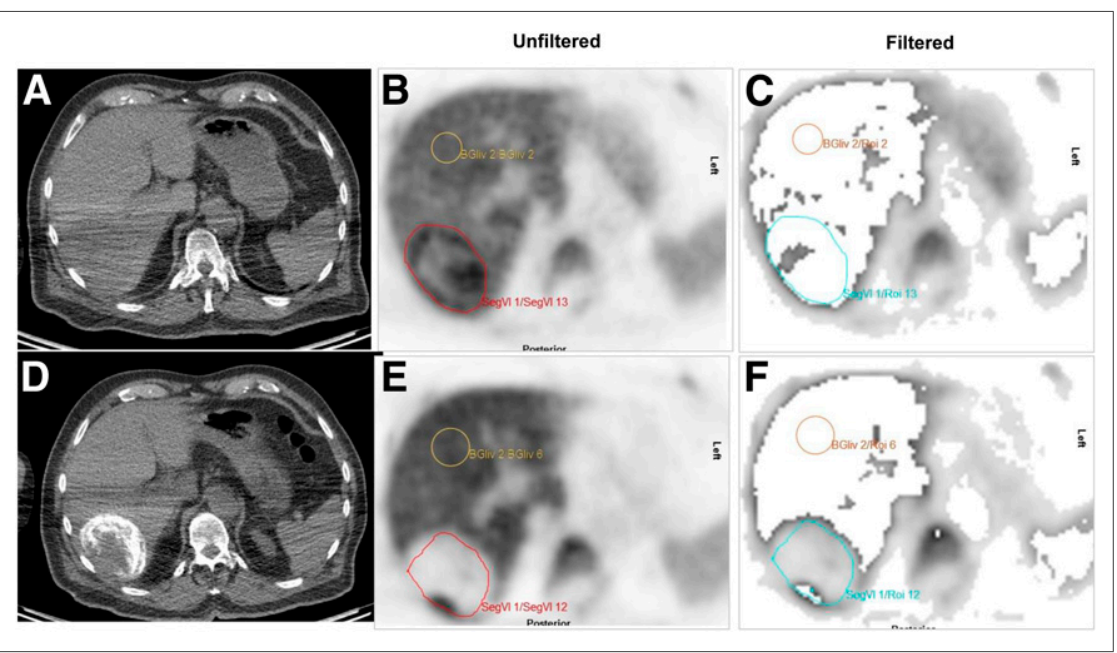

FIGURE 2. Axial CT, unfiltered ${ }^{18} \mathrm{~F}$-FLT PET, and filtered ${ }^{18} \mathrm{~F}$-FLT PET images before $(\mathrm{A}-\mathrm{C})$ and after (D-F) TACE show focal HCC lesion (red outline) with increased uptake at baseline and reduction after TACE. (B and E) In one of two baseline PET images before application of KSF (B), HCC is visible above background. (C) After application of KSF, tumor is mostly filtered out. (E) In post-TACE images, HCC is photopenic compared with surrounding liver before application of KSF. (F) After application, background liver activity is removed and $\mathrm{HCC}$ remains visible. BGliv = background liver; Seg = segment. Yellow outline indicates background liver, and blue outline indicates focal HCC lesion on following application of KSF.

\section{DISCUSSION}

There is still no single tracer recommended by international guidelines for either diagnosis or response assessment in HCC (1). The main limitation of the studied tracers has been a poor tumor-tobackground liver ratio resulting in a dual-tracer approach for visualizing $\mathrm{HCC}$, which is time-consuming and exposes patients to significant radiation (5). We hypothesized that because ${ }^{18} \mathrm{~F}$-FLT uptake is specific for tumor proliferation, tracer uptake will not be affected by the presence of inflammation (16). Moreover, we investigated the utility of KSF to improve visualization of HCC by removing background liver activity.

To address the hypothesis that tumor ${ }^{18} \mathrm{~F}-\mathrm{FLT}$ uptake will change predictably with effective treatment, we first assessed the messenger RNA expression of factors responsible for handling ${ }^{18} \mathrm{~F}-\mathrm{FLT}$. TYMS catalyzes the last step in the de novo synthesis of thymidine monophosphate (TMP), whereas $T K-1$ catalyzes synthesis of TMP via the salvage pathway. $T K-1$ affects ${ }^{18}$ F-FLT cellular trapping and is a surrogate marker of proliferation (17). Moreover, we have shown that with TYMS inhibition, ${ }^{18}$ F-FLT uptake increases because of redistribution of the membrane transporter SLC29A1 to the plasma membrane (18). Expression levels of TYMS, $T K-1$, and $S L C 29 A 1$ were all markedly upregulated in HCC compared with normal liver; we confirmed marked upregulation in protein expression of $T K-1$ in HCC compared with surrounding matched cirrhotic tissue consisting of both regenerative and dysplastic nodules, suggesting that ${ }^{18} \mathrm{~F}$-FLT could be useful in differentiating HCC from surrounding cirrhotic tissue.

Our prospective ${ }^{18}$ F-FLT PET study illustrated that most intrahepatic lesions had increased tracer uptake consistent with the tissue microarray findings. To improve HCC visualization, we applied the previously validated $\operatorname{KSF}(19,20)$. However, fewer lesions were detected with KSF than with standard PET/CT imaging; most small lesions-those smaller than $3 \mathrm{~cm}$-were 
TABLE 2

Baseline Dynamic PET Parameters $(n=14)$

\begin{tabular}{ccccccc}
\hline Patient no. & $K_{1}(\mathrm{~mL} / \mathrm{min} / \mathrm{g})$ & $k_{2}(1 / \mathrm{min})$ & $k_{3}(1 / \mathrm{min})$ & $k_{4}(1 / \mathrm{min})$ & $V_{\mathrm{b}}(\mathrm{mL} / \mathrm{g})$ & $K_{\mathrm{i}}(\mathrm{mL} / \mathrm{min} / \mathrm{g})$ \\
\hline 1 & 0.16 & 0.10 & 0.10 & 0.11 & $5.0 \mathrm{E}-0$ & 0.08 \\
2 & 0.17 & 0.076 & 0.074 & 0.07 & 0.015 & 0.09 \\
3 & 0.31 & 0.40 & 0.24 & 0.04 & 0.019 & 0.11 \\
4 & 0.21 & 0.28 & 0.13 & 0.06 & 0.034 & 0.07 \\
5 & 0.34 & 0.17 & 0.097 & 0.04 & 0.03 & 0.13 \\
6 & 0.43 & 0.28 & 0.086 & 0.01 & 0.058 & 0.10 \\
8 & 0.282 & 0.28 & 0.13 & 0.02 & 0.04 & 0.09 \\
9 & 0.29 & 0.53 & 0.32 & 0.04 & 0.05 & 0.11 \\
11 & 0.41 & 0.51 & 0.17 & 0.02 & 0.07 & 0.10 \\
13 & 0.11 & 0.16 & 0.04 & 0.001 & 0.10 & 0.02 \\
14 & 0.46 & 0.42 & 0.13 & 0.03 & $2.9 \mathrm{E}-04$ & 0.11 \\
15 & 0.58 & 0.69 & 0.13 & 0.02 & 0.07 & 0.09 \\
16 & 0.26 & 0.18 & 0.08 & 0.02 & 0.07 & 0.08 \\
18 & 0.23 & 0.19 & 0.09 & 0.02 & $8.0 \mathrm{E}-06$ & 0.07 \\
\hline
\end{tabular}

filtered out. One possible explanation is that the KSF compares voxel temporal profiles with standard tissue profiles and that both liver and lesion voxel profiles were highly variable in our group. Another explanation is that the cirrhotic background has a high relative uptake that, in combination with the profile variability, reduces the ability of the KSF to effectively discriminate HCC from the proliferating, background, liver. A third possible explanation is that partial-volume effects may contribute to filtering out of small lesions.

When considering ${ }^{18} \mathrm{~F}-\mathrm{FLT}$ PET imaging for lesion detection, our findings are in keeping with those of Eckel et al., who reported a 72\% sensitivity for ${ }^{18} \mathrm{~F}$-FLT in visualizing HCC, with a similar median SUV and tumor-to-liver ratios (10). Similar sensitivities have been reported for ${ }^{11} \mathrm{C}$-acetate PET and for a dual-tracer approach with ${ }^{18} \mathrm{~F}-\mathrm{FDG}$ and ${ }^{11} \mathrm{C}$-acetate, $75 \%$ and $73 \%$, respectively $(21,22)$. However, Ho et al. reported single-tracer sensitivity of $87 \%$ for ${ }^{11} \mathrm{C}$-acetate, increasing to $100 \%$ sensitivity using 2 tracers (23). These differences in diagnostic sensitivity may be a reflection of the subgroup analysis undertaken in the study of Ho et al. Overall, the fact that the literature does suggest improved diagnostic sensitivity using a dual-tracer approach motivates the development of alternate tracers for the detection of HCC.

We investigated the role of dynamic ${ }^{18} \mathrm{~F}-\mathrm{FLT}$ PET as a predictor of TACE response. The radiologic response to TACE was $54 \%$ by mRECIST and $60 \%$ by PET, with good concordance between imaging modalities. Cascales-Campos et al. considered using ${ }^{18} \mathrm{~F}$-FDG PET to assess response to TACE before liver transplantation (24). The authors described a reduction in ${ }^{18} \mathrm{~F}$ FDG uptake correlating with the degree of necrosis on pathologic examination of the explanted liver, and other investigators have considered minimum SUV cutoffs for defining response to therapy (25). In a retrospective study, Park et al. investigated the utility of a dual-tracer approach with ${ }^{18} \mathrm{~F}-\mathrm{FDG}$ and ${ }^{11} \mathrm{C}$-acetate in predicting response to TACE (7). They did not investigate concordance between the imaging modalities but observed that the ratio of ${ }^{18} \mathrm{~F}$-FDG to ${ }^{11} \mathrm{C}$-acetate predicted response to TACE, determining a cutoff from ROC analysis. We selected a $20 \%$ reduction in $\mathrm{SUV} 60_{\max }$ to indicate response, a cutoff extrapolated from breast cancer studies (26). Larger studies are needed to define a more accurate cutoff for HCC than was possible to derive from our small data set.

A key strength of this study is that dynamic PET imaging allows us to establish the basis of the PET signal change in HCC, considering that TACE has an acute impact on blood flow. In line with our hypothesis, we report a significant reduction in $K_{1}$ after TACE, illustrating an abrupt reduction in tissue perfusion. This finding is in sharp contrast to antiangiogenic chemotherapy, for which an increase in $K_{1}$ due to vessel normalization and reduced interstitial pressure can accompany response (27). Dynamic studies using ${ }^{11} \mathrm{C}$-acetate reported a reduced $K_{1}$ in HCC lesions supplied by the hepatic artery, compared with benign lesions supplied by the portal vein, because the radiotracer concentration time-course is initially delayed as the portal flow passes through the splanchnic circulation (28). Huo et al. reported that because of the differential time course of radiotracers circulating through the hepatic artery or portal vein, lesions supplied by the hepatic artery will reach a stable concentration of radiotracer earlier and at a higher peak after injection; hence, arterialized lesions will have a lower $K_{1}$ than benign lesions that are supplied predominantly by the portal vein (29). $K_{\mathrm{i}}$ is related to the phosphorylation of thymidine in the tissue (30). $K_{\mathrm{i}}$ has previously been found to correlate with ${ }^{18} \mathrm{~F}$-FLT SUV (31), and our results illustrate a similar correlation. Moreover, we report baseline $K_{\mathrm{i}}$ and $\mathrm{V}_{\mathrm{b}}$ to be predictive of TACE response to treatment, suggesting that responding tumors are more actively proliferating and have higher perfusion suggestive of higher vascularity.

Study limitations include a small sample size and a lack of correlation between PET uptake parameters and histology. If subjects have whole-body imaging, full assessment of extrahepatic disease could be performed; the correlation between PET kinetic parameters and SUV means that future whole-body static imaging would be supported. In addition, manual VOIs could not be used to determine thresholds because components of the tumor were close to background liver activity. In addition, some lesions became isointense or photopenic relative to background on follow-up PET imaging. 


\section{CONCLUSION}

We have shown that $T K-1$ expression is significantly higher in HCC than in surrounding cirrhotic tissue, supporting the use of ${ }^{18}$ F-FLT PET. High ${ }^{18}$ F-FLT PET uptake was seen in most HCC tumors; however, application of KSF did not improve visualization because of the high and variable SUV both in tumors and in background cirrhotic liver. Imaging of proliferation with ${ }^{18} \mathrm{~F}-\mathrm{FLT}$ PET could be used to predict response to TACE in this small case series. Although this study was a pilot study, the results generated are provocative and should be taken forward to larger prospective trials correlating with outcome.

\section{DISCLOSURE}

This study was supported by the Imperial College NIHR Imperial Biomedical Research Centre and the Imperial Experimental Cancer Medicines Centre, Cancer Research U.K. (C2536/A16584). The views expressed are those of the authors and not necessarily those of the NIHR or the Department of Health and Social Care. No other potential conflict of interest relevant to this article was reported.

\section{ACKNOWLEDGMENTS}

We thank Kasia Kowlozski, Robert Punjani, Hope McDevitt, and Vijay Vaja for conducting the study.

\section{KEY POINTS}

QUESTION: Does dynamic ${ }^{18}$ F-FLT PET allow accurate visualization of HCC?

PERTINENT FINDINGS: ${ }^{18} \mathrm{~F}-\mathrm{FLT}$ PET can differentiate HCC from surrounding cirrhotic tissue, with PET parameters correlating with TACE response.

IMPLICATIONS FOR PATIENT CARE: ${ }^{18 F-F L T ~ P E T ~ c a n ~ a c c u-~}$ rately detect $\mathrm{HCC}$ and should be further investigated, particularly for assessment of response.

\section{REFERENCES}

1. European Association for the Study of the Liver. EASL clinical practice guidelines: management of hepatocellular carcinoma. J Hepatol. 2018;69: 182-236.

2. Chapman WC, Majella Doyle MB, Stuart JE, et al. Outcomes of neoadjuvant transarterial chemoembolization to downstage hepatocellular carcinoma before liver transplantation. Ann Surg. 2008;248:617-625.

3. Gordic S, Corcuera-Solano I, Stueck A, et al. Evaluation of HCC response to locoregional therapy: validation of MRI-based response criteria versus explant pathology. J Hepatol. 2017;67:1213-1221.

4. Kim S, Mannelli L, Hajdu CH, et al. Hepatocellular carcinoma: assessment of response to transarterial chemoembolization with image subtraction. J Magn Reson Imaging. 2010;31:348-355.

5. Bieze M, Klumpen HJ, Verheij J, et al. Diagnostic accuracy of ${ }^{18}$ F-methylcholine PET/CT for intra- and extrahepatic hepatocellular carcinoma. Hepatology. 2014;59:996-1006.

6. Weber G, Morris HP. Comparative biochemistry of hepatomas. III. Carbohydrate enzymes in liver tumors of different growth rates. Cancer Res. 1963;23: 987-994.

7. Park S, Kim TS, Kang SH, Kim HB, Park JW, Kim SK. ${ }^{11}$ C-acetate and ${ }^{18}$ Ffluorodeoxyglucose positron emission tomography/computed tomography dual imaging for the prediction of response and prognosis after transarterial chemoembolization. Medicine (Baltimore). 2018;97:e12311.

8. Toyohara J, Waki A, Takamatsu S, Yonekura Y, Magata Y, Fujibayashi Y. Basis of FLT as a cell proliferation marker: comparative uptake studies with $\left[{ }^{3} \mathrm{H}\right]$ thymidine and $\left[{ }^{3} \mathrm{H}\right]$ arabinothymidine, and cell-analysis in 22 asynchronously growing tumor cell lines. Nucl Med Biol. 2002;29:281-287.
9. van Waarde A, Jager PL, Ishiwata K, Dierckx RA, Elsinga PH. Comparison of sigma-ligands and metabolic PET tracers for differentiating tumor from inflammation. J Nucl Med. 2006;47:150-154.

10. Eckel F, Herrmann K, Schmidt S, et al. Imaging of proliferation in hepatocellular carcinoma with the in vivo marker ${ }^{18} \mathrm{~F}$-fluorothymidine. J Nucl Med. 2009;50: 1441-1447.

11. Gray KR, Contractor KB, Kenny LM, et al. Kinetic filtering of $\left[{ }^{18} \mathrm{~F}\right]$ fluorothymidine in positron emission tomography studies. Phys Med Biol. 2010;55:695709.

12. Pinato DJ, Ramachandran R, Toussi ST, et al. Immunohistochemical markers of the hypoxic response can identify malignancy in phaeochromocytomas and paragangliomas and optimize the detection of tumours with VHL germline mutations. Br J Cancer. 2013;108:429-437.

13. Lencioni R, Llovet JM. Modified RECIST (mRECIST) assessment for hepatocellular carcinoma. Semin Liver Dis. 2010;30:52-60.

14. Kenny L, Coombes RC, Vigushin DM, Al-Nahhas A, Shousha S, Aboagye EO. Imaging early changes in proliferation at 1 week post chemotherapy: a pilot study in breast cancer patients with $3^{\prime}$-deoxy- $3^{\prime}-\left[{ }^{18} \mathrm{~F}\right]$ fluorothymidine positron emission tomography. Eur J Nucl Med Mol Imaging. 2007;34:1339-1347.

15. Contractor K, Challapalli A, Tomasi G, et al. Imaging of cellular proliferation in liver metastasis by $\left[{ }^{18} \mathrm{~F}\right]$ fluorothymidine positron emission tomography: effect of therapy. Phys Med Biol. 2012;57:3419-3433.

16. van Waarde A, Cobben DC, Suurmeijer AJ, et al. Selectivity of ${ }^{18} \mathrm{~F}-\mathrm{FLT}$ and ${ }^{18} \mathrm{~F}-$ FDG for differentiating tumor from inflammation in a rodent model. $\mathrm{J} \mathrm{Nucl} \mathrm{Med}$. 2004;45:695-700.

17. Kenny LM, Vigushin DM, Al-Nahhas A, et al. Quantification of cellular proliferation in tumor and normal tissues of patients with breast cancer by $\left[{ }^{18} \mathrm{~F}\right]$ fluorothymidine-positron emission tomography imaging: evaluation of analytical methods. Cancer Res. 2005;65:10104-10112.

18. Perumal M, Pillai RG, Barthel H, et al. Redistribution of nucleoside transporters to the cell membrane provides a novel approach for imaging thymidylate synthase inhibition by positron emission tomography. Cancer Res. 2006;66:8558-8564.

19. Challapalli A, Barwick T, Pearson RA, et al. $3^{\prime}$-deoxy- $-3^{\prime}-{ }^{18}$ F-fluorothymidine positron emission tomography as an early predictor of disease progression in patients with advanced and metastatic pancreatic cancer. Eur J Nucl Med Mol Imaging. 2015;42:831-840.

20. Contractor K, Challapalli A, Tomasi G, et al. Imaging of cellular proliferation in liver metastasis by $\left[{ }^{18} \mathrm{~F}\right]$ fluorothymidine positron emission tomography: effect of therapy. Phys Med Biol. 2012;57:3419-3433.

21. Li S, Peck-Radosavljevic M, Ubl P, et al. The value of $\left[{ }^{11} \mathrm{C}\right]$-acetate PET and $\left[{ }^{18} \mathrm{~F}\right]-\mathrm{FDG}$ PET in hepatocellular carcinoma before and after treatment with transarterial chemoembolization and bevacizumab. Eur J Nucl Med Mol Imaging. 2017;44:1732-1741.

22. Park JW, Kim JH, Kim SK, et al. A prospective evaluation of ${ }^{18} \mathrm{~F}-\mathrm{FDG}$ and ${ }^{11} \mathrm{C}$ acetate PET/CT for detection of primary and metastatic hepatocellular carcinoma. J Nucl Med. 2008;49:1912-1921.

23. Ho CL, Yu SC, Yeung DW. ${ }^{11} \mathrm{C}$-acetate PET imaging in hepatocellular carcinoma and other liver masses. J Nucl Med. 2003;44:213-221.

24. Cascales-Campos PA, Ramirez P, Lopez V, et al. Prognostic value of 18-fluorodeoxyglucose-positron emission tomography after transarterial chemoembolization in patients with hepatocellular carcinoma undergoing orthotopic liver transplantation. Transplant Proc. 2015;47:2374-2376.

25. Lee JW, Yun M, Cho A, et al. The predictive value of metabolic tumor volume on FDG PET/CT for transarterial chemoembolization and transarterial chemotherapy infusion in hepatocellular carcinoma patients without extrahepatic metastasis. Ann Nucl Med. 2015;29:400-408.

26. Kenny L, Coombes RC, Vigushin DM, Al-Nahhas A, Shousha S, Aboagye EO. Imaging early changes in proliferation at 1 week post chemotherapy: a pilot study in breast cancer patients with $3^{\prime}$-deoxy- $3^{\prime}-\left[{ }^{18} \mathrm{~F}\right]$ fluorothymidine positron emission tomography. Eur J Nucl Med Mol Imaging. 2007;34:1339-1347.

27. Scarpelli M, Simoncic U, Perlman S, Liu G, Jeraj R. Dynamic ${ }^{18}$ F-FLT PET imaging of spatiotemporal changes in tumor cell proliferation and vasculature reveals the mechanistic actions of anti-angiogenic therapy. Phys Med Biol. 2018;63:155008.

28. Winterdahl M, Keiding S, Sorensen M, Mortensen FV, Alstrup AK, Munk OL. Tracer input for kinetic modelling of liver physiology determined without sampling portal venous blood in pigs. Eur J Nucl Med Mol Imaging. 2011;38:263-270.

29. Huo L, Guo J, Dang Y, et al. Kinetic analysis of dynamic ${ }^{11} \mathrm{C}$-acetate PET/CT imaging as a potential method for differentiation of hepatocellular carcinoma and benign liver lesions. Theranostics. 2015;5:371-377.

30. Muzi M, Spence AM, O'Sullivan F, et al. Kinetic analysis of $3^{\prime}$-deoxy-3'- ${ }^{18} \mathrm{~F}$ fluorothymidine in patients with gliomas. J Nucl Med. 2006;47:1612-1621.

31. Muzi M, Vesselle H, Grierson JR, et al. Kinetic analysis of 3'-deoxy-3'-fluorothymidine PET studies: validation studies in patients with lung cancer. $J$ Nucl Med. 2005;46:274-282. 